\title{
A Novel Design of Analog Signal Power Amplifier Module for Relay Protection Tester
}

\author{
Jianshi Bai ${ }^{1, a,{ }^{*}}$, Tong $\mathrm{Wu}^{1, \mathrm{~b}}$, Fang Shen ${ }^{1, \mathrm{c}}$, Yi Liang $^{1, \mathrm{~d}}$, Jing Gao ${ }^{1, \mathrm{e}}$, Zhentao \\ $\operatorname{Han}^{1, f}$ \\ ${ }^{1}$ Economic Research Institute of State Grid Liaoning Electric Power Co.,Ltd., Shenyang, Liaoning, \\ China \\ alunaticbjs89@126.com
}

Keywords: Analog Signal Power Amplifier Module, Rely Protection Tester.

\begin{abstract}
The analog signal power amplifier module is an important component of relay protection tester. As is known, most of the analog signal power amplifier modules employ single output loops to achieve the output accuracy regardless of the output current, which may lead to large capacity, low efficiency and more cost. In order to overcome these deficiencies aforementioned, a novel design for the analog signal power amplifier module is proposed in this paper. This design improves the complementary push-pull current amplification circuit, and adds the logical control loop and the analog switch so that the current amplification circuit can switch different output loops according to the output current, which results in lower capacity, volume and cost, and higher efficiency. Finally, the new analog power amplifier module shows good amplitude-frequency property in the simulation and has ability to meet the relay protection tester demand under different frequency.
\end{abstract}

\section{Introduction}

The analog signal power amplifier module of relay protection tester is composed of voltage amplifier and current amplifier. Its output power through $\mathrm{D} / \mathrm{A}$ conversion is too low to test relay protection device directly. Thus, performance indicators of the power amplifier would badly influence the accuracy of the relay protection tester. The improved analog signal current amplification circuit of relay protection tester is stated from design standpoint in this paper. The structure of the power amplifier module of relay protection tester is shown in Figure 1.

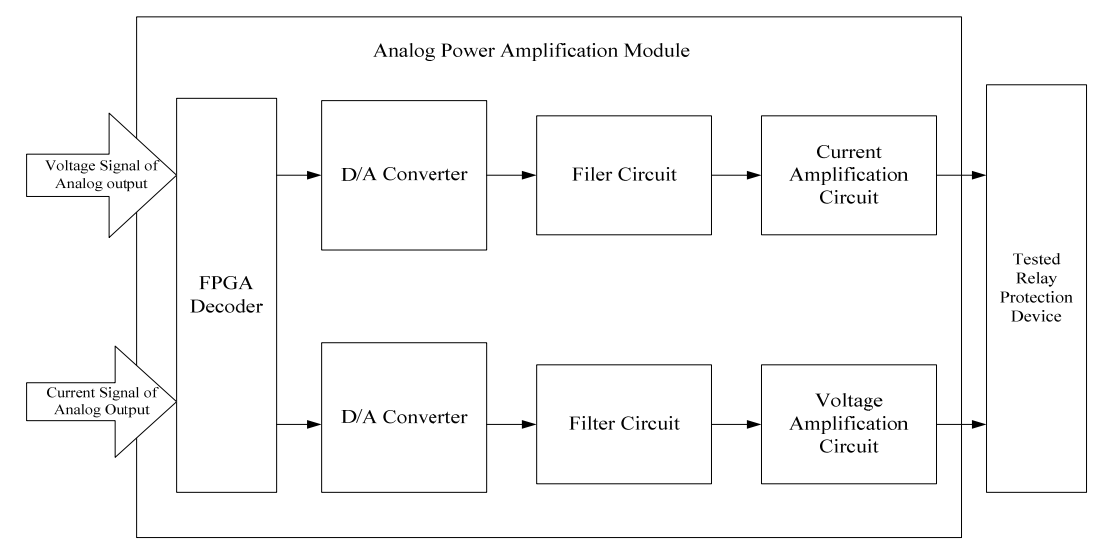

Fig.1 The structure of the analog signal power amplifier module of relay protection tester

Though Class-D AMP is mature, relay protection tester cannot choose Class-D AMP for several reasons. Firstly, its low output bandwidth cannot meet the demand of relay protection tester. Secondly, Class-D AMP generates crossover distortion easily, and its fidelity is lower than Class-A AMP and Class-B AMP so that the Class-D AMP cannot meet the high fidelity demand of relay protection tester. Thirdly, Class-D AMP cannot amplify high frequency harmonics with high fidelity for now, but relay protection tester need to amplify power-frequency component and high frequency harmonics with high fidelity. Finally, Class-D AMP with power electronic devices has large volume which is different from the small and portable feature of relay protection tester. 
Most of the analog signal power amplifier modules apply single output loop regardless of the output current to achieve the output accuracy. Therefore, two points must be noticed when choosing the output circuit resistance for the power amplifier of relay protection tester. One is that output accuracy cannot be guaranteed if the resistance is too small. The other is that the power amplifier will have lower efficiency, larger capacity and higher cost if the resistance is too large.

Based on the above problems, a new design of the analog signal power amplification circuit is proposed.

\section{Design of Current Amplification Circuit}

\subsection{The Theory of Current Amplification Circuit Design}

The Power amplification circuit are commonly divided into two types, transformer coupling push-pull and complementary push-pull. The transformer coupling push-pull one has narrow bandwidth, heavy weight, and the shank saturation may cause non-linear distortion. However, the complementary push-pull one has many advantages on current amplification circuit, such as wide bandwidth, little volume, light weight, low cost, low distortion, high efficiency and amplification without distortion. Thus, the complementary push-pull circuit is chosen as the fundamental form for amplification circuit. The current amplification circuit is shown in Figure 2.

Input stage is constituted by operational amplifier LF356 because it can reduce the input dislocate, adjust bias current and bias voltage, and adjust zero potentiometer to make the potential of the output stage neutral point zero while no input signal.

Input stage link is the driver that is constituted by triode $\mathrm{T} 1$ and $\mathrm{T} 3$. When collector of $\mathrm{T} 3$ is forward, T4, T5 are conducting. When collector of T3 is negative, T6, T7 are conducting.

A constant-current power supply constituted by triode T2, resistance R6, R7 and R8 is used for stabilizing bias current which changes with the signal.

Diode D1, D2 and D3 are used for giving feeble forward bias to output stage which made the circuit on Class-AB work state when amplification circuit running. To choose Class-AB work state for amplification circuit is because Class-AB amplification circuit can not only overcome the low efficiency of Class A amplification circuit, but also avoid the crossover distortion of class B amplification circuit.

The complementary Darlington circuit is constituted by triode T4, T5 and T6.

Mosfet MOS1 and MOS2 are NMOS. Drain of MOS1 as the input connects the emitter of T5, source as output connects resistance R15 and the load, and grid connects logic control loop. Drain of MOS2 as input connects the emitter of T7, source as output connects resistance R15 and the load, and grid connects logic control loop.

The cut-off of NMOS is controlled by logic control loop. When the output current of current amplifier is larger than or equal to 15A, grid voltage reaches threshold, and current flow past NMOS which is close. At this time, the analog switch change supper circuit which is without accumulator carriage to breakover. When the output current of current amplifier is smaller than 15A, grid voltage is under the threshold, and NMOS is open so that current flow past sample resistance R16. At this time, the analog switch change upper circuit which has accumulator carriage to breakover.

The parallel current negative feedback loop is constituted by Mosfet MOS1, MOS2, resistance R16, R17, R15, R2 and logic control loop, analog switch, accumulator carriage. Above those, R15 which is $0.02 \Omega, \mathrm{R} 16$ which is $0.3 \Omega, \mathrm{R} 17$ which is $0.3 \Omega$ are sample resistances. The feedback voltage is proportional to output current, and parallels to LF356 after dividing voltage with R2 to forms the current feedback loop. The main reason to use current negative feedback loop is that current negative feedback has the same effect on AC and DC and the same strong stability.

Therefore, this new power amplifier operate under the current which is smaller than $15 \mathrm{~A}$ with $\pm 20 \mathrm{~V}$ DC source for most time which reduces power cost due to the frequently switch between Mosfet and analog switch so that the efficiency of the new power amplifier can be improved.

When designing the new amplifier, these three key points need to be particularly noticed which will be discussed in this paper:The improved power amplification circuit with theory and simulation 
test's effects on the primary power amplification circuit.The design of logic control circuit, especially the set of the starting voltage.The control of sequence, which is that the change of the analog switch must be after logic control loop changes Mosfet and precise and quick.

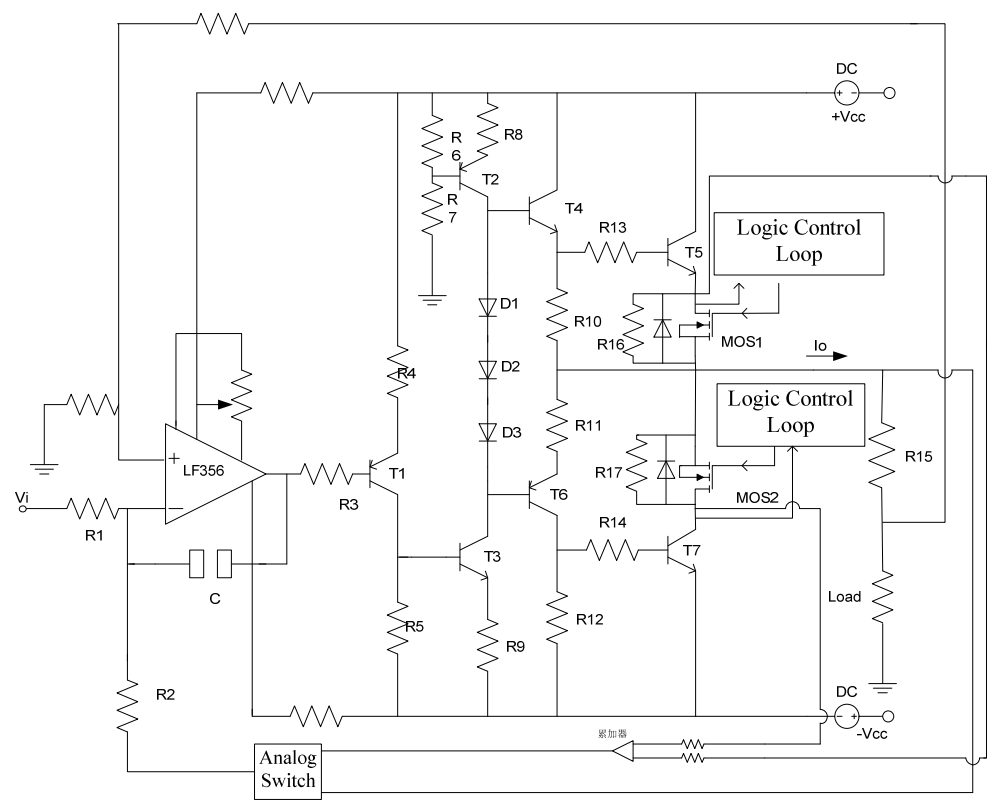

Fig.2 The New Current Amplification Circuit

\subsection{Effect of R16 and R17 on Amplifier Circuit}

The amplifier circuit is shown in Fig.2, the maximum output current of the circuit will be 50A if R16 and R17 are not included in the sampling circuit. We can analyze the effect of the sampling resistors of R16 and R217 on the maximum output current. The static operating point UceQ5 of T5 is $10 \mathrm{~V}$, which can be calculated by the original data, and then the maximum output current can be estimated to be $20 \mathrm{~A}$ according to the static operating point UceQ5.

The threshold current of the amplifier circuit is set to be 15A, MOS1 and MOS2 will be in the off-state when the output current od the circuit is smaller than $15 \mathrm{~A}$, the current goes though the branch including R16 and R17. When the output current is larger than $15 \mathrm{~A}$, the current will go through the branch of MOS1 and MOS2, which are in the on-state, and the output current achieves the maximum value of 50A.

Therefore, resistors R16 and R17 have no effect on the amplification gain of the circuit, which can be secured to operate normally all the time.

\subsection{Design of the Logic Control Circuit}

The operating principle of the logic control circuit as shown in Fig.3 will be detailed taking the positive half axis of the power amplifier circuit, where the voltage source is $5 \mathrm{~V}$.

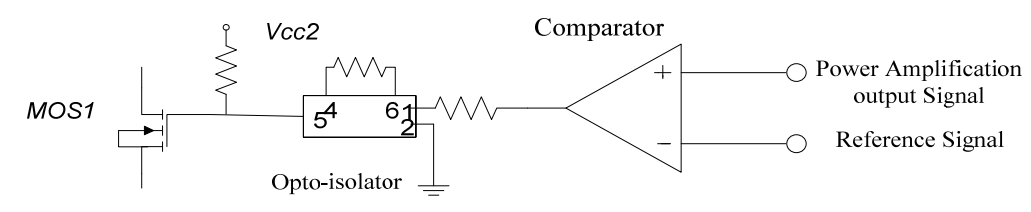

Fig.3 Diagram of the logic control circuit

The negative input port of the comparator is the reference voltage, and the positive input port will be the output signal of the power amplifier circuit. Comparing the two inputs, if the output signal of the power amplifier circuit is larger than that of the reference signal, then the comparator will output a low voltage level, and a high output level for the photoelectric isolating device. The level will be sent to MOS1 and makes it on the conduction status. The current may pass the MOS1 branch, analog switch connects the lower circuit (without a accumulator)to conduct the pass. On the contrary, if the output signal of the power amplifier circuit is smaller than that of the reference signal, a high voltage level will bethe output for the comparator, and low voltage level for the 
photoelectric isolating device, then MOS1 switches off and the current goes though the R16 branch, the analog switch controls the upper arm (with a accumulator)to conduct.

\subsection{Sequential Switch of Analog Switch}

Analog switch is in charge of the switching transients of the feedback control circuit, the switch will take action to conduct the lower arm when the output current of amplifier circuit is larger than or equal to $15 \mathrm{~A}$ and vice versus. The logic control signal gets feedback from the output circuit of the amplifier. Much more attention should be paid to the sequence of switching transients, which should lag the switching-on of MOSFET.

\subsection{Analysis of the Current Source Feature of Current Amplification Circuit}

The equivalent electric circuit of a novel power amplifier is shown in Fig.4 and Fig.5 respectively. The following part will focus on the current source characterization of the novel power amplifier based on the equivalent electric circuit.

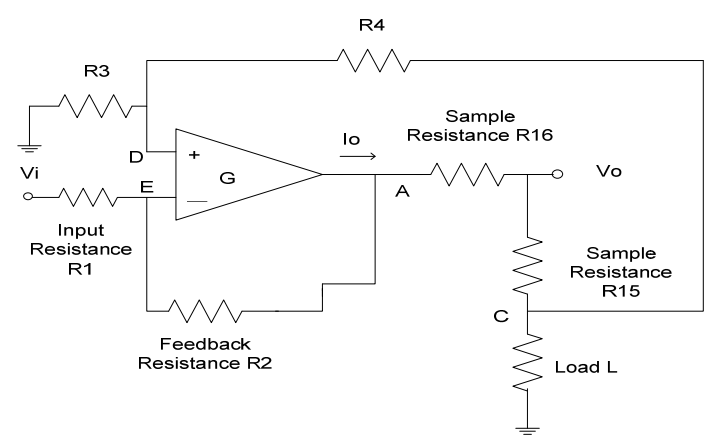

Fig.4 The Equivalent Circuit of the new power amplification circuit when output current is smaller than $15 \mathrm{~A}$

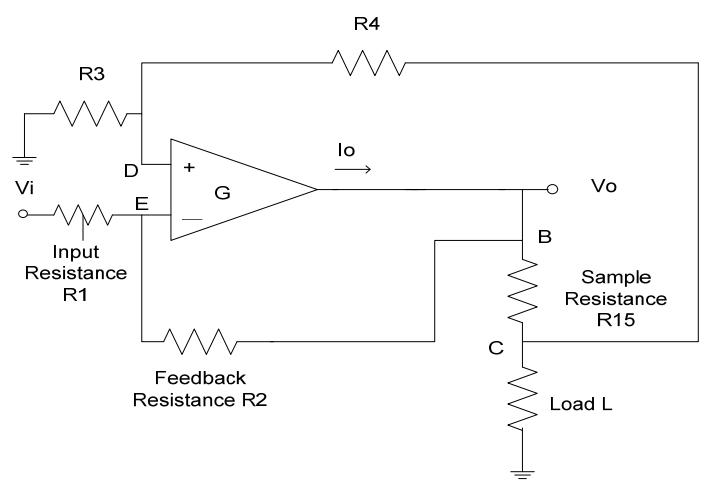

Fig.5 The Equivalent Circuit of the new power amplification circuit when output current is larger than or equal to $15 \mathrm{~A}$

Nodal point equation can be acquired according to the equivalent circuit of the power amplifier in Fig 4

$$
\begin{gathered}
\left(G_{1}+G_{2}\right) \times V_{E}-G_{1} \times V_{i}-G_{2} \times V_{A}=0 \\
\left(G_{3}+G_{4}\right) \times V_{D}-G_{4} \times V_{C}=0 \\
\frac{G_{16} \times G_{15}}{G_{16}+G_{15}}+G_{4}+G_{L} \times V_{C}-\frac{G_{16} \times G_{15}}{G_{16}+G_{15}} \times V_{A}-G_{4} \times V_{D}=0
\end{gathered}
$$

The following relationship can be obtained based on some basics of electric circuit

$$
\begin{gathered}
V_{D}-V_{E}=0 \\
I_{\mathrm{L}}=V_{\mathrm{C}} \times G
\end{gathered}
$$

Substituting (2-4) and (2-5) into (2-2), (2-3), (2-1) and (2-5) one has

$$
I_{\mathrm{L}}=\frac{G_{\mathrm{L}} G_{1} G_{4} G_{15} G_{16}\left(G_{3}+G_{4}\right)}{G_{1 \mathrm{l}}^{2} G_{15} G_{16}\left(G_{1}+G_{2}\right)-\left(G_{55} G_{\mathrm{L}}+G_{4} G_{15}+G_{16} G+G_{4} G_{16}+G_{4} G_{15} G_{1}\right) \times\left(G_{3}+G_{4}\right) G_{2}+G_{2} G_{4}^{3}\left(G_{15}+G_{16}\right)} Y_{i}
$$


The resistances in Fig. 4 are $\mathrm{R} 1=\mathrm{R} 3$ and $\mathrm{R} 2=\mathrm{R} 4$. Change conductance in (2-6) into resistance form, and then one obtains

$$
I_{\mathrm{L}}=\frac{R_{1}+R_{2}}{R_{1}+R_{2}+R_{\mathrm{L}}} \times \frac{R_{2}}{R_{1} \times\left(R_{15}+R_{16}\right)} V_{i}
$$

The load resistance in the amplification circuit is much smaller than $\mathrm{R} 1$ and $\mathrm{R} 2$, thus it can be ignored. The equation (2-7) can be rewritten as

$$
I_{L}=\frac{R_{2}}{R_{1} \times\left(R_{15}+R_{16}\right)} V_{i}
$$
5

Nodal point equation can be got according to the equivalent circuit of the power amplifier in Fig

$$
\begin{gathered}
\left(G_{1}+G_{2}\right) \times V_{E}-G_{1} \times V_{i}-G_{2} \times V_{A}=0 \\
\left(G_{3}+G_{4}\right) \times V_{D}-G_{4} \times V_{C}=0 \\
G_{15}+G_{4}+G_{\text {负 }}^{--} \times V_{C}-G_{15} \times V_{A}-G_{4} \times V_{D}=0
\end{gathered}
$$

The calculation here is similar to above for Fig. 4 , and the equation of amplification can be derived as

$$
I_{\mathrm{L}}=\frac{R_{2}}{R_{1} \times R_{15}} V_{i}
$$

According to the equations (2-8) and (2-12), it can be seen that the load current is only related to the R1,R2 and the sampling resistors(R15 or R15+R16) no matter the output current of the novel power amplifier is smaller or larger than $15 \mathrm{~A}$ and has nothing with the load. Also, the load current is proportional to the input voltage, which shows excellent current source characteristic.

\section{Simulation Validation of the Current Amplifying Circuit}

This section will analyze the performance of the power amplifier by means of amplitudefrequency characteristic test, current source characteristic test, input voltage and output current waveform comparison test and switching circuit waveform test under different frequency.

\subsection{Amplitude-Frequency Characteristic Test of the Current Amplifying Circuit}

Take two input voltages, which make the output current greater than $15 \mathrm{~A}$ and less than $15 \mathrm{~A}$ respectively, and keep the input voltage constant. Then we change the input signal frequency, and get the data as shown in Table 1.

Table 1 Magnitude-frequency Characteristic of Current Amplifier

\begin{tabular}{|c|c|c|c|}
\hline Frequency(Hz) & $\leq 20 \mathrm{~Hz}$ & $20 \sim 2000 \mathrm{~Hz}$ & $2000 \sim 10000 \mathrm{~Hz}$ \\
\hline Input Current & $20.0 \mathrm{~A}$ & $20.0 \mathrm{~A}$ & $20.0 \mathrm{~A}$ \\
\hline Output Current & $3.0 \mathrm{~A}$ & $3.0 \mathrm{~A}$ & $3.0 \mathrm{~A}$ \\
\hline
\end{tabular}

Conclusions can be get from the table that the upper frequency of the novel amplifier circuit is far greater than the standard requirements of the amplifier circuit when the current is greater than $15 \mathrm{~A}$ and less than $15 \mathrm{~A}$, and the waveform is smooth and flawless. Therefore, the new amplifier circuit meets the design requirements of amplitude-frequency.

\subsection{Test of the Effect on Circuit Switch under Different Frequency}

Change the Vi frequency of the current amplifying circuit shown in Figure 2. taking a certain input voltage value of three intervals: less than $20 \mathrm{~Hz}, 20$ to $2000 \mathrm{~Hz}, 2000$ to $10000 \mathrm{~Hz}$ for example respectively, then we can detect the output current waveform changes in the process of switching, as shown in Figure 6.

\subsection{Current Waveform Contrast Test between Input Voltage and Current}

Table 2 are derived according to input and output parameters, and the waveforms are shown in Fig. 7. 


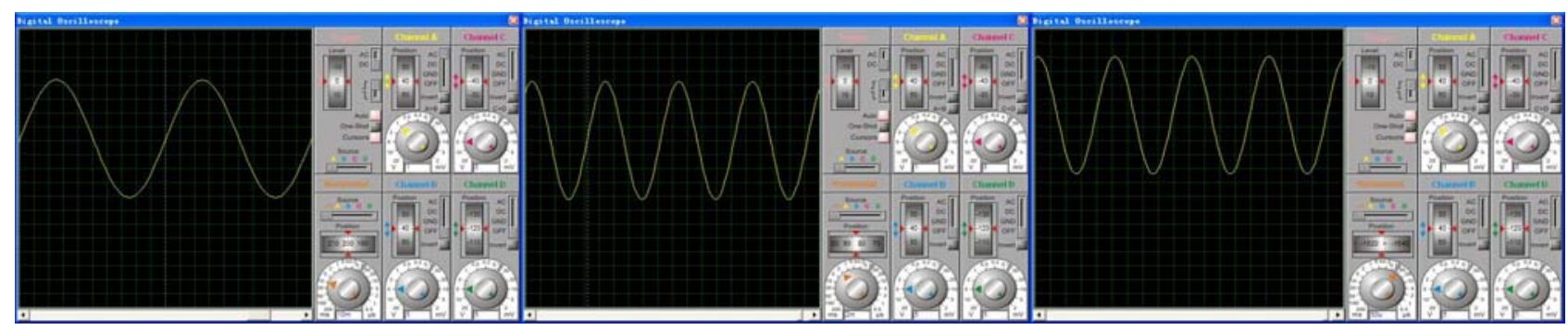

Fig 6 Input waveform of change process of output current under $20 \mathrm{~Hz} / 20 \sim 2000 \mathrm{~Hz} / 2000 \sim 10000 \mathrm{~Hz}$

Table 2 Contrast between input voltage and output current

\begin{tabular}{|c|c|c|c|c|}
\hline & Input Voltge & Output Current & Input Voltage & Output Current \\
\hline Virtual Value & $10 \mathrm{~V}$ & $20 \mathrm{~A}$ & $15 \mathrm{~V}$ & $30 \mathrm{~A}$ \\
\hline Frequency & $50 \mathrm{~Hz}$ & $50 \mathrm{~Hz}$ & $50 \mathrm{~Hz}$ & $50 \mathrm{~Hz}$ \\
\hline Multiple & 2 Times & 2 Times & 2 Times & 2 Times \\
\hline
\end{tabular}

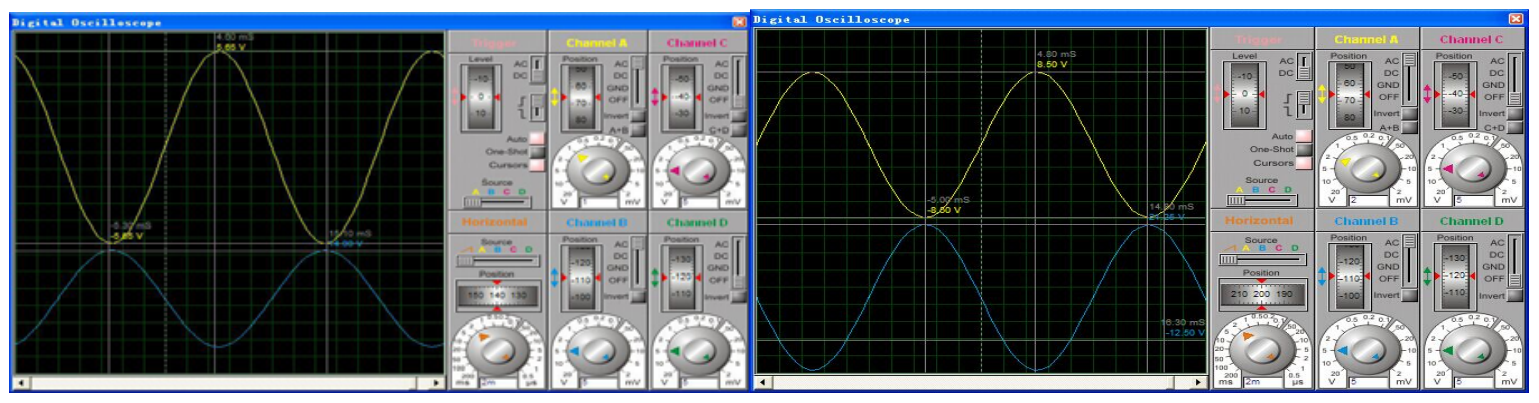

Fig.7 Waveform of $10 \mathrm{~V}$ and $15 \mathrm{~V}$ input voltage and 20A output current

\section{Conclusion}

In this paper, a novel analog power amplifier module has been designed for the relay protection testers. The amplification module is made up of a current amplifier circuit using a complementary push-pull type. The current amplifying circuit realizes the function of switching the output circuit of the different current amplifying circuit according to the output current. The current amplification circuit has obvious advantages over the original current amplification circuit and D-type power amplifier through the theoretical analysis and simulation results. The maximum output power can be reduced by up to $60 \%$ compared with the original amplifier circuit. The maximum power reduction reduces volume of the power amplifier, power supply and protection circuit correspondingly, resulting in a one-third reduction in the actual size of the tester's analog power amplifier module. It is obvious that the new design of the power amplifier circuit can reduce the maximum power and size, save the cost and improve the efficiency of the design purpose for the power amplifier module.

\section{References}

[1] Harrison R R. (2002) A low-power low-noise CMOS amplifier for neural recording applications[C]. IEEE International Symposium on Circuits and Systems, IEEE Xplore, 197-200.

[2] Zhi-Heng Xu, Liu J W,Xiang Q. (2004)Some key problems about microcomputer based relay protection tester[J]. Relay,33(11), 12-18.

[3] Qin Zhou, Meimei Hu, Kexu Chen. (2012) Research and Development of the Detection Device for Tester for Tester of Relay Protection[J]. Process Automation Instrumentation,33(4), 52-54.

[4] Lijun Qin. (1987) Research on Computerized Modern Protection Test Device[D]. North China Electric Power University, Bei Jing.

[5] Fei Dong. (1992) A new method of relay protection test-hardware development of power system simulator [D].North China Electric Power University, Bei Jing. 